\title{
Comparison of Implicit and Symbolic Implicit Monte Carlo Line Transport with Frequency Weight Vector Extension
}

M.S. McKinley, E.D. Brooks III, A. Szoke

December 3, 2002

U.S. Department of Enargy

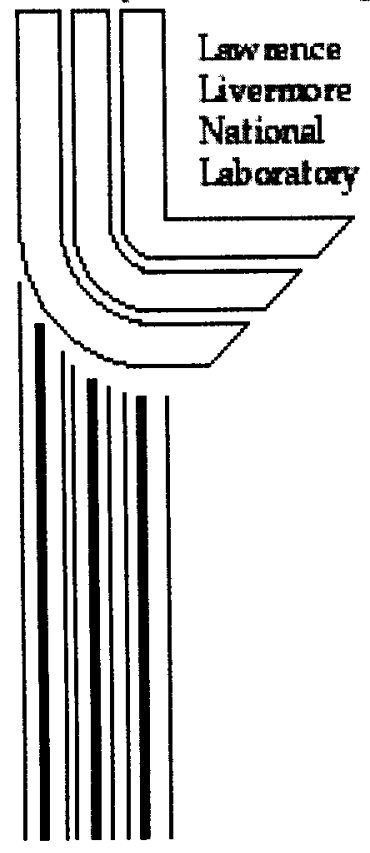




\section{DISCLAIMER}

This document was prepared as an account of work sponsored by an agency of the United States Government. Neither the United States Government nor the University of California nor any of their employees, makes any warranty, express or implied, or assumes any legal liability or responsibility for the accuracy, completeness, or usefulness of any information, apparatus, product, or process disclosed, or represents that its use would not infringe privately owned rights. Reference herein to any specific commercial product, process, or service by trade name, trademark, manufacturer, or otherwise, does not necessarily constitute or imply its endorsement, recommendation, or favoring by the United States Government or the University of California. The views and opinions of authors expressed herein do not necessarily state or reflect those of the United States Government or the University of California, and shall not be used for advertising or product endorsement purposes.

This work was performed under the auspices of the U. S. Department of Energy by the University of California, Lawrence Livermore National Laboratory under Contract No. W-7405-Eng-48.

This report has been reproduced directly from the best available copy.

Available electronically at http://www.doc.gov/bridge

Available for a processing fee to U.S. Department of Energy

And its contractors in paper from

U.S. Department of Energy

Office of Scientific and Technical Information

P.O. Box 62

Oak Ridge, TN 37831-0062

Telephone: (865) 576-8401

Facsimile: (865) $576-5728$

E-mail: reports@adonis.osti.gov

Available for the sale to the public from

U.S. Department of Commerce

National Technical Information Service

5285 Port Royal Road

Springfield, VA 22161

Telephone: (800) 553-6847

Facsimile: (703) 605-6900

E-mail: orders@ntis.fedworld.gov

Online ordering: http://www.ntis.gov/ordering.htm

OR

Lawrence Livermore National Laboratory

Technical Information Department's Digital Library

http://www.llnl.gov/tid/Library.html 


\title{
Comparison of Implicit and Symbolic Implicit Monte Carlo Line Transport with Frequency Weight Vector Extension
}

\author{
Michael Scott McKinley Eugene D. Brooks III Abraham Szoke \\ University of California \\ Lawrence Livermore National Laboratory \\ Livermore, California 94550 \\ UCRL-ID-147898
}

\begin{abstract}
We compare the Implicit Monte Carlo (IMC) technique to the Symbolic IMC (SIMC) technique, with and without weight vectors in frequency space, for timedependent line transport in the presence of collisional pumping. We examine the efficiency and accuracy of the IMC and SIMC methods for test problems involving the evolution of a collisionally pumped trapping problem to its steady-state, the surface heating of a cold medium by a beam, and the diffusion of energy from a localized region that is collisionally pumped. The importance of spatial biasing and teleportation for problems involving high opacity is demonstrated. Our numerical solution, along with its associated teleportation error, is checked against theoretical calculations for the last example.
\end{abstract}

Key words: Monte Carlo, symbolic, photon, transport, teleportation error, X Ray Laser

PACS: $42.55 . \mathrm{Vc}, 95.30 . \mathrm{Jx}, 95.30 . \mathrm{Ky}$

\section{Introduction}

Time-dependent transport of radiation from resonance lines involving spontaneous emission is used in the study of stellar atmospheres and in laser produced plasmas. The transport equation for photons is coupled to a time-dependent level population equation. This system of equations can prove difficult to solve

Email addresses: mckinley901lnl.gov (Michael Scott McKinley), brooks301lnl.gov (Eugene D. Brooks III), szoke101lnl.gov (Abraham Szoke). 
due to stiffness and the wide range of opacity inherent in an atomic line profile. Deterministic methods, known as complete linearization and accelerated lambda iteration[1], remain the methods of choice for applications in stellar atmospheres [2]. Nevertheless, Monte Carlo methods for radiation transport are still of more than historical interest. They can be valuable for X-ray laser applications and problems involving fast time variation and geometrical complexity not easily addressed by deterministic methods. In this paper we study test problems of simple geometry but of significant time dependence.

Advances have been made in the area of Monte Carlo methods for this problem. Encouraged by the success and robustness of implicit Monte Carlo techniques in local thermodynamic equilibrium, an implicit method was developed for line transport. While Implicit Monte Carlo (IMC)[3] works very well for most cases, it contains an effective scattering term that is inefficient when dealing with optically thick problems and that becomes negative for an atomic line in the gain regime. The Symbolic Implicit Monte Carlo (SIMC) method [4] was born in the notion that you can track and score spontaneously emitted particles with an unknown symbolic weight that is determined at the end of a time step. This removes the source of the ineffiency, the effective scattering term, from the IMC method.

The extension of the SIMC method to thermal radiation in local thermodynamic equilibrium was first published in Ref. [5]. A key feature of that work was noting that, once effective scattering is removed, one may track particles containing weight vectors in frequency space instead of using statistical sampling. Little demonstration of the advantage of weight vectors is offered in Ref. [5]. We demonstrate in this paper that a significant advantage results if one desires spectral information from the problem output.

The stability and accuracy of the IMC and SIMC methods have been thoroughly analyzed by N'Kaoua and Sentis [6], for the case of linear transport. Their analysis applies directly to the line transport problems considered in this paper.

The goal of this paper is to compare the three methods (IMC, SIMC and SIMC with weight vectors in frequency space) for slab geometry. The codes that implement these methods model a two level atom in slab geometry with collisional coupling between levels and with incident radiation. We study the methods for a variety of problems in an attempt to compare and contrast the techniques under a wide range of conditions. We look at computational efficiency, accuracy and convergence of results as a function of time step, zone size and variable width zoning strategies. Sensitivity to spatial biasing for problems involving high opacity, which can have a significant impact on computational efficiency, is also explored. 
Three test problems of study are presented in this paper. The first problem examines the performance of the methods for high opacity. The second test problem studies an opaque cold slab heated by monochromatic incident radiation that is off line center. The third test problem looks at a case where the only source of photons is a central zone in a slab that is collisionally pumped. This test problem has a partial analytical solution that is used to verify the accuracy of the numerical methods.

In this investigation, we demonstrate that the SIMC method does well in reducing noise for high opacity problems, and that the weight vector extension to SIMC provides a further substantial reduction in noise for problems where spectral information is desired. We also show that a geometric progression of zone sizes near an interface, with spatial biasing for spontaneous emission, is effective in improving the performance of these methods. Finally, we show that IMC is less susceptible to teleportation error than SIMC, but that this advantage evaporates as the time step size is reduced in order to obtain better temporal accuracy.

\section{Mathematical Method}

\subsection{Derivation of Methods}

For a two-level system in slab geometry that includes collisional pumping between atomic levels, the radiation transport equation is

$$
\frac{\partial f}{\partial t}+\mu c \frac{\partial f}{\partial x}=\frac{n_{2}}{2} A_{21} \phi-c\left(K_{12} n_{1}-K_{21} n_{2}\right) \phi f
$$

where $c$ is the speed of light, $x$ is the position in the slab, $\mu$ is the direction cosine of the radiation, $\nu$ is the frequency of the radiation, $f(\mu, \nu, x, t)$ is the photon number density distribution per unit atom density, $n_{2}(x, t)$ is the upper level population fraction, $n_{1}(x, t)$ is the lower level population fraction, $A_{21}$ is the spontaneous emission rate, $\phi(a, \nu)$ is the Voigt line profile normalized to unit integral [7], and $K_{12}=\kappa N$ where $\kappa$ is the lower state absorption cross section and $N$ is the atom number density. The coefficient $K_{21}$ is defined by

$$
K_{21}=\frac{g_{1}}{g_{2}} K_{12}
$$

where $g_{1}$ and $g_{2}$ are the statistical weights for levels 1 and 2, respectively. As in [3], we consider the problem in the regime of complete redistribution and no physical scattering of photons. 
The equations governing the atomic population fractions $n_{1}$ and $n_{2}$ are

$$
\begin{aligned}
\frac{d n_{2}}{d t}= & C_{12} n_{1}-C_{21} n_{2}-A_{21} n_{2}+c\left(K_{12} n_{1}-K_{21} n_{2}\right) \\
& \times \int_{-1}^{1} d \mu \int_{0}^{\infty} d \nu \phi(\nu) f(\mu, \nu)
\end{aligned}
$$

and

$$
n_{1}+n_{2}=1
$$

where $C_{12}$ and $C_{21}$ are rate constants for the collisional transitions $1 \rightarrow 2$ and $2 \rightarrow 1$, respectively. One must also add in appropriate boundary conditions and initial state to the above equations.

Using (4), equations (1) and (3) may be rewritten as

$$
\frac{\partial f}{\partial t}+\mu c \frac{\partial f}{\partial x}=\frac{n}{2} A_{21} \phi-c\left[K_{12}-\left(K_{21}+K_{12}\right) n\right] \phi f
$$

and

$$
\begin{aligned}
\frac{d n}{d t}= & C_{12}-\left(C_{12}+C_{21}+A_{21}\right) n+c\left[K_{12}-\left(K_{21}+K_{12}\right) n\right] \\
& \times \int_{-1}^{1} d \mu \int_{0}^{\infty} d \nu \phi(\nu) f(\mu, \nu)
\end{aligned}
$$

respectively, where $n$ is the upper level population fraction.

We can generate a finite differencing scheme in time for (6) by using the standard IMC technique [3]. We integrate (6) from $t_{0}$ to $t_{0}+\Delta t$. In the spontaneous emission and collision terms, we approximate $n(t)$ by $n\left(t_{0}+\Delta t\right)$. In the absorption term, we substitute $n\left(t_{0}\right)$ for $n(t)$ and obtain

$$
\begin{aligned}
n\left(t_{0}+\Delta t\right)= & n\left(t_{0}\right)+\left[C_{12}-\left(C_{12}+C_{21}+A_{21}\right) n\left(t_{0}+\Delta t\right)\right] \Delta t \\
& +c\left[K_{12}-\left(K_{21}+K_{12}\right) n\left(t_{0}\right)\right] \\
& \times \int_{t_{0}}^{t_{0}+\Delta t} d t \int_{-1}^{1} d \mu \int_{0}^{\infty} d \nu \phi(\nu) f(\mu, \nu, t) .
\end{aligned}
$$


In the standard IMC technique [3], we substitute $n\left(t_{0}+\Delta t\right)$ from (7) into the spontaneous emission term of (5) while using $n\left(t_{0}\right)$ in the absorption term. After some algebra, we obtain

$$
\begin{aligned}
\frac{\partial f}{\partial t}+\mu c \frac{\partial f}{\partial x}= & \frac{\gamma A_{21} \phi}{2}\left[n\left(t_{0}\right)+\Delta t C_{12}\right]+\frac{\gamma c A_{21} \phi \Delta t}{2} \\
& \times\left[K_{12}-\left(K_{21}+K_{12}\right) n\left(t_{0}\right)\right] \int_{-1}^{1} d \mu \int_{0}^{\infty} d \nu \phi(\nu) f(\mu, \nu) \\
& -c \phi\left[K_{12}-\left(K_{21}+K_{12}\right) n\left(t_{0}\right)\right] f,
\end{aligned}
$$

where $\gamma$ is defined as

$$
\gamma=\frac{1}{1+\Delta t\left(C_{12}+C_{21}+A_{21}\right)}
$$

Equation (8) can be interpreted as a transport equation with a net absorption, $\sigma_{a}$, and effective scattering, $\sigma_{s}$, contribution as given by

$$
\sigma_{a}(\nu)=\hat{f} \phi(\nu)\left[K_{12}-\left(K_{21}+K_{12}\right) n\left(t_{0}\right)\right]
$$

and

$$
\sigma_{s}(\nu)=(1-\hat{f}) \phi(\nu)\left[K_{12}-\left(K_{21}+K_{12}\right) n\left(t_{0}\right)\right]
$$

where the fraction, $\hat{f}$, is given by

$$
\hat{f}=\frac{1+\Delta t\left(C_{12}+C_{21}\right)}{1+\Delta t\left(C_{12}+C_{21}+A_{21}\right)}
$$

The fraction, $\hat{f}$, determines how much effective scattering the problem contains. As $\hat{f}$ approaches unity, effective scattering vanishes. The disadvantage of this method is that the effective scattering term dominates the execution time in optically thick problems, resulting in very long problem runs. Reducing the effective scattering by making $\hat{f}$ approach 1 requires a smaller time step which also increases execution time and may reduce accurracy (see Sec. 2.5).

Symbolic Implicit Monte Carlo [4] achieves implicit time integration using a different point of view. Photons produced by spontaneous emission are given a symbolic weight that remains undetermined until the end of the time step. The Monte Carlo procedure is the same as for IMC, except there is no effective scattering term. Particle scoring results in a linear system of equations that are solved for the upper atomic population fraction at the end of a time step. After 
spatial discretization (see Sec. 2.3), the upper atomic population is updated with

$$
\begin{aligned}
n\left(t_{0}+\Delta t\right)_{i}= & n\left(t_{0}\right)_{i}+\left[C_{12}-\left(C_{12}+C_{21}+A_{21}\right) n\left(t_{0}+\Delta t\right)_{i}\right] \Delta t \\
& +c\left[K_{12}-\left(K_{21}+K_{12}\right) n\left(t_{0}\right)_{i}\right] \\
& \times\left[F N_{i}+\sum_{j} F S_{i j} n\left(t_{0}+\Delta t\right)_{j}\right] / V_{i}
\end{aligned}
$$

The quantities in the square brackets are short hand for the following integral

$$
\int d x \int_{t_{0}}^{t_{0}+\Delta t} d t \int_{-1}^{1} d \mu \int_{0}^{\infty} d \nu \phi(\nu) f(\mu, x, \nu, t) .
$$

In Eq. (13), the term containing $F N_{i}$ is the contribution to the change in upper state population within zone $i$ from photons that were present at the beginning of the time step. They have therefore explicit, numerical weights. The term containing $F S_{i j} n\left(t_{0}+\Delta t\right)_{j}$ is a similar contribution coming from bundles with symbolic weights that were born in zone $j$ during the time step; $n\left(t_{0}\right)_{i}$ is the upper level atomic population fraction in zone $i$ at start of time step; $n\left(t_{0}+\Delta t\right)_{i}$ is the unknown upper atomic population fraction in zone $i$ at the end of the time step; and $V_{i}$ is the thickness of zone $i$. For further details, we refer to Ref. [4]. Although the problems we present will involve small numbers of zones, and as a result the solution of small linear systems using a direct solver, there is some concern that the size of the linear system to be solved will become intractable using direct solution methods for problems with larger zone counts. Jacobi iteration [8], or other more sophisticated techniques, do very well at solving the system of equations given in (13) by using the values in the previous time step for the initial starting point.

\subsection{Weight Vector Extension}

Since SIMC removes effective scattering, it has a simpler Monte Carlo simulation process than that of standard IMC. While the frequency (energy) of a photon may alter how its weight is attenuated due to differing absorption cross sections, it does not alter the photon's direction. If there is no real physical scattering, two photons with the same geometric starting conditions, differing only in frequency, will traverse the same path through the problem. This offers the possibility of treating frequency space deterministically by selecting a frequency discretization and associating a weight vector, indexed by this discretization, with the photon. 
As was done in Ref. [5], we have extended the SIMC algorithm by associating a vector of weights, indexed by the frequency group, for each photon simulated. Instead of sampling the line profile for spontaneously emitted photons as is the case for IMC, this method constructs the vector of weights by assigning to each emission frequency the birth weight times the emission probability for that frequency. Unlike Ref. [5], we do not collapse the weight vector to a single frequency at the end of time step. Instead, we carry the vector of weights through successive time steps, obtaining deterministic spectral information when the particle leaves the problem domain.

The weight vector approach, in the absence of frequency dependent physical scattering (e.g. Compton Scattering), handles frequency space deterministically. The role of Monte Carlo is then relegated to integrating the possibly complicated geometry of the problem. This approach has a significant advantage over frequency sampling when the frequencies with a high emission probability are also strongly absorbed, with photons being transported elsewhere in the frequency spectrum. An example, demonstrated in this paper, is the case of line transport with a high opacity at line center where most of the transport occurs in the wings of the line. Using the weight vector approach, every photon samples the important frequency region where transport occurs and develops the correct output spectrum for the geometrical path being sampled.

\subsection{Uniform and Geometric Zoning}

We use a finite differencing method where the upper atomic population, $n(x, t)$, is defined at the zone center, and for any time step, $n(x, t)$ is constant within each zone. We have tried two basic zoning schemes: equally spaced zones and spacing based on a geometric progression starting from the surface, where the thickness of the surface zone was close to one optical depth at line center. Figure 1 shows the whole problem divided into 7 zones where $a$ is the smallest zone, $b$ is the length of the slab, and $\beta$ is a multiplicative factor. Only the solid lines are to be considered; the dashed lines lines show a subzone scheme that will be discussed later. We have found that this geometric spacing is essential to accommodate the boundary layer: a rapid change of the atomic population with depth near the surface and a rapid development of the spectrum of photons leaving the surface. The SIMC method was more sensitive to zoning than the IMC method due to the portion of the absorption that is transmuted into effective scattering in IMC, but the same issues prevail in the limit of small time step size in IMC.

When the problem of interest involves high opacity, little transport occurs at line center. The emission profile is strongly peaked at line center but so is the 


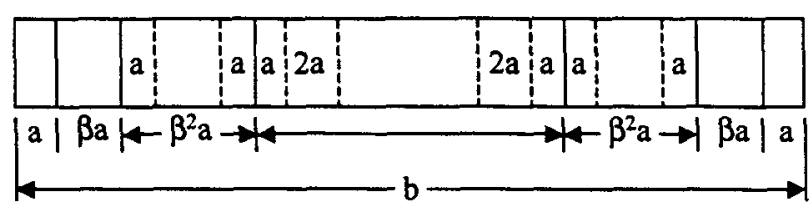

Fig. 1. Geometric Zone and Subzone Construction

absorption profile. As a result the more rarely produced frequencies (far from line center) travel further and account for much of the transport. This property of line transport (strongly emitted frequencies are also strongly absorbed) makes optically thick problems quite sensitive to zoning and importance sampling schemes. Straightforward Monte Carlo sampling of the emission profile, only to have these photons absorbed, produces results with excessive noise.

\subsection{Biasing}

The first spatial importance sampling scheme we have investigated is directly tied to the zoning. For a problem with high opacity at line center, the small zones near the surface are very important to getting the transport and emission spectrum right, and therefore must be treated with more particles per unit volume than the thicker zones in the center of the problem. We have compared three schemes. The first is straightforward emission of particles with a constant density per unit volume (unbiased). The next scheme involves emission of an equal number of particles in each zone, which translates to a geometric weighting (based on the zoning scheme), in favor of the smaller zones near the surface. The final scheme involves a further geometric biasing within each zone so particles are born closer to interfaces where they have a greater chance of crossing between zones.

Favoring of photons born near the surface of an opaque problem is key to obtaining an emission spectrum with low statistical error. For photons at line center, only those born near the surface have a good chance of streaming out of the problem and making a contribution to the output spectrum. Importance sampling schemes improve the statistics of escaping photons that have a significant weight in the line center frequencies.

A final step in importance sampling is required to efficiently treat the large central zones in problems with high line center opacity. If particles are emitted uniformly within the zone, one emits line center photons with high probability. These photons travel only a short distance within the zone before their weight shrinks to the point of being insignificant, and their particle history is terminated. A lot of computer time is expended computing a deterministic 
equilibrium solution for the central zone, while getting very noisy results for the transport across zone boundaries, the quantity of interest. We found that just as the geometric zoning scheme with an equal number of photons in each zone improves the emission spectrum from a surface, a similar geometric subzone scheme to importance sample the regions near the surface of the interior zones reduces the noise in the transport between thick interior zones, resulting in an improved solution throughout the problem.

The subzone biasing scheme is very similar to the zone biasing described above. One creates a subzone grid for each real zone in the problem, starting with a thin subzone at each surface of an interior zone. Successive subzone sizes are then obtained in a geometric progression by increasing the subzone size by a factor of 2 , working inwards to the interior of the zone from both sides. The process is stopped when the remainder of the zone is just larger than the subzone on each side of it. In Fig. 1, we show this subzone configuration using the dashed lines. Just as was the case for zonal biasing, an equal number of photons is emitted in each subzone of a given zone. The weights of the photons emitted in each subzone are adjusted so that emitted weight is distributed uniformly across the zone and the correct total weight is emitted within the zone.

We would like to note that one could employ directional biasing for photons born near a zone interface, favoring those heading in the direction of the interface; as that would contribute to transport between zones. We do not explore this in this paper but would expect an advantage to be generated for SIMC where there is no effective scattering. For IMC, the effective scattering term would produce angular mixing that would remove the advantage for this type of biasing.

\subsection{Photon Teleportation}

The equations governing the atomic populations are discretized in space and time, and one can expect some systematic errors to arise from this in a scheme where atomic populations are constant within a zone. Photon teleportation is an error that arises when the absorption mean free path is small compared to the size of a zone, and the time step size is also small (in the case for IMC). As photons stream through a zone they are attenuated by absorption. If the opacity is high, photons entering one side of a zone are completely absorbed very near the boundary. The scoring of energy deposition, however, is done as if the absorption had taken place evenly across the entire zone. On the next time step, the corresponding spontaneous emission is handled assuming this uniformity in the zone. In effect, spontaneous emission occurs too early on the far side of the zone and energy is transported across the problem too quickly. 
This error is aggravated in IMC if the time step size is reduced without a coordinated adjustment to the zone size.

SIMC is much more susceptible to this problem than IMC. This is due to the reduced net absorption for IMC, where a portion of the physical absorption has been converted to effective scattering. This beneficial influence of effective scattering comes at the cost of increased execution time and evaporates as the time step size is reduced. In the limit that $\Delta t$ goes to 0, IMC has the same teleportation error as SIMC. Teleportation error is properly addressed by decreasing the optical depth per zone. Unfortunately, reduction of zone size in order to reduce teleportation error leads to an increased running time as particles cross many zones in order to escape the problem.

Photon teleportation has been observed in many previous studies. In fact, it is known that a finite difference discretization that assumes constant conditions within each zone and time step does not tend to the diffusion limit of the transport equation [6,9-14]. A heuristic treatment informally known as source tilting [15], frequently used in IMC, could be used in SIMC to reduce teleportation effects. A self consistent, piecewise linear treatment of atomic population would obtain the correct diffusion limit.

\section{Example Problems}

We use three test problems to probe the properties of the implicit Monte Carlo methods studied in this paper. The first example is a simple collisionally pumped system used in Refs. [3] and [4], although we push to a much higher opacity given the increased speed of modern microprocessors. The second example models the heating of cold media by incident radiation. The third example, which has an analytical solution available for comparison purposes, involves collisional pumping in the center of the slab.

In comparing the methods, the two issues of concern are systematic and statistical errors. The systematic error is an error in the numerical modeling of the physics that persists in the limit of large Monte Carlo particle count. It can be controlled by suitably refining the time step size, the choice of zones, the choice for frequency bins, or by modifying a given method to be higher order accurate in these parameters. The implicit transport methods examined in this paper develop subtle interplay between these different controls on discretization error, and we will demonstrate this in the results presented below.

Given the discretization parameters that control systematic error (and any importance sampling scheme) the number of Monte Carlo particles controls 
the statistical error for a given problem run. In the limit of large particle count, the statistical error scales inversely with the square root of the particle count. This fact provides a means to evaluate the relative efficiency of the methods. We choose a particle count for each method that results in a given execution time (first confirming that the noise envelope, in fact the standard deviation of individual runs, is scaling like the square root of the particle count in each case) and then examine the noise envelope for 100 independent runs. The relative speeds of the methods, in the limit of large particle count, are then given as the square of the ratios of the measured noise envelopes. We would like to note that while this is a good way to compare the relative efficiency of the methods under examination, we do not suggest that this is how users should employ Monte Carlo applications in practice.

Unless otherwise stated, each example will use 16 frequency groups, each of 0.2 Doppler widths. In addition, the line profile, and therefore the frequency spectrum, is symmetric around zero and we take advantage of this symmetry. Spatially, each problem is divided up into 21 equally spaced zones unless noted otherwise.

In the results below, problem output is always presented as the mean and standard deviation (not error in the mean) of 100 independent problem runs. The mean of a large number of problem runs provides the best opportunity to spot systematic error, while the standard deviation gives us a good idea of just how much scatter would be present in a single run along with a reliable way to estimate the computational efficiency of the method.

\subsection{Collisionally Pumped Trap}

The first example studied is a slab of unit width that is collisionally pumped and has a steady-state optical depth of 1000 . The slab is uniform with equally spaced zones and has no incident radiation impinging on it. The problem input parameters, $K_{12}$ and $K_{21}$, were tuned in order to obtain the specified optical depth at line center. See Table 1 for the physical parameters. The pumping started at $t=0$ and the problem was run until steady-state was reached.

The main difference between IMC and SIMC is that IMC has effective scattering while in SIMC photons quickly stream through the problem and feel the full toll of absorption. Since relatively small changes, as a function of the position coordinate, are occurring in the middle of the slab while a high rate of change exists near the boundaries, it makes sense to examine the effects of zone refinement and spatial biasing. We will first examine geometric zoning without biasing by making the zone widths near the boundary thinner using a geometric progression, with a ratio, $\beta=1.75$, for adjacent zones as discussed 
Table 1

Physical Parameters for Collisionally Pumped Trap Example

\begin{tabular}{cc}
\hline Parameter & Value \\
\hline$n(x, t=0)$ & 0 \\
$f(\mu, \nu, x, t=0)$ & 0 \\
$f(\mu>0, \nu, x=0, t)$ & 0 \\
$f(\mu<0, \nu, x=1, t)$ & 0 \\
Voigt Parameter & 0.0 \\
$K_{12}$ & 2170 \\
$K_{21}$ & 2170 \\
$A_{21}$ & 3.3 \\
$C_{12}$ & 0.25 \\
$C_{21}$ & 0.67 \\
\hline
\end{tabular}

in Section 2.3. Smaller zones are needed to resolve the atomic population near the surface of the slab where the basic assumption of the numerical method, that the atomic population is constant within the zone, is violated.

The upper atomic population is shown in Fig. 2 for the uniformly zoned case as well as for the geometrically zoned case. Only the first tenth of the slab is shown as the upper level atomic population does not change much past what is displayed. In this figure, it is clear that geometric zoning is needed to properly capture the shape of the upper level atomic population near the surface. To check the correctness of the numerical solution, we have estimated the atomic population near the center of the slab with an escape probability analysis[7] and have found that it agrees our Monte Carlo solution.

In Fig. 3, we show the standard deviation for the 100 problem instances used to produce the averaged results shown in Fig. 2. The noise envelope for SIMC is about a factor of 6 less than that for IMC on this problem, corresponding to a factor of 36 improvement in execution speed. Geometric zoning, without a compensating importance sampling, has increased the noise envelope near the border and has reduced it away from the surface, due to the relatively few particles born in the smaller zones.

The exit spectrum for the collisionally pumped trap example is plotted in Fig. 4 as a function of frequency in units of Doppler widths for the geometrically zoned scheme. Uniform zoning is not shown as it is very poor due to teleportation errors as discussed in Sec. 2.5. While the uniform zone spectrum (not shown) for IMC is very noisy, it does give the correct frequency output shape since its effective scattering prevents photon teleportation. This figure 


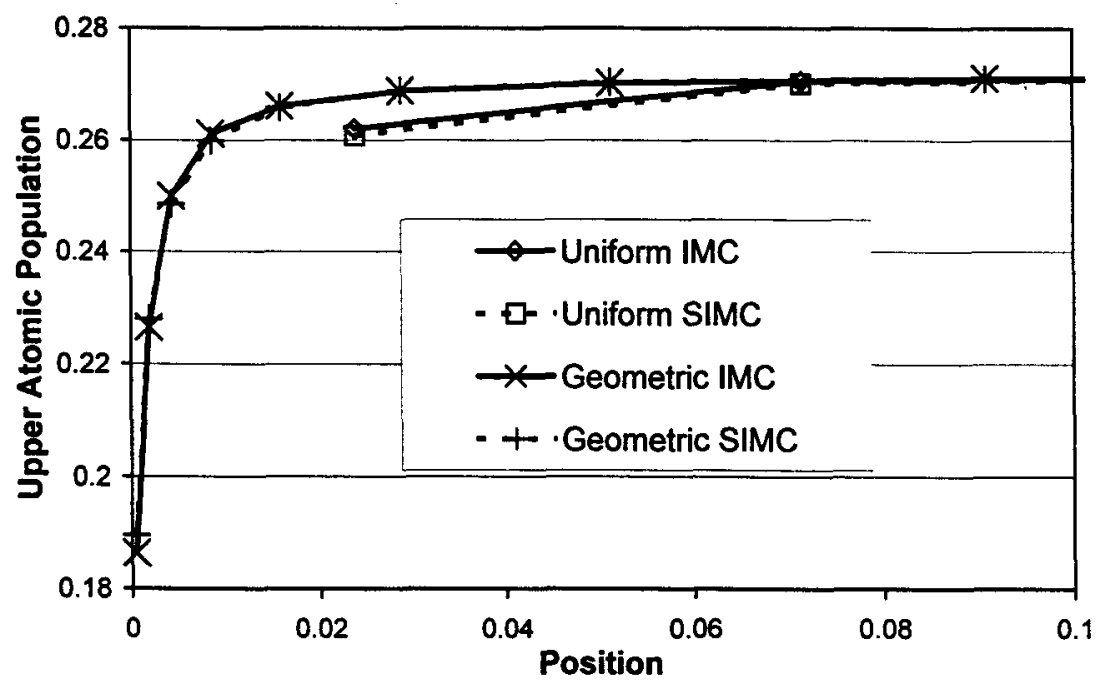

Fig. 2. Upper Atomic Population for Collisionally Pumped Trap Example

shows that all three methods predict the same exit frequency distribution when geometrically zoned. IMC's plot is less accurate due to noise which will be discussed next.

When examining the noise envelope for the frequency dependent output, as given in Fig. 5, SIMC with weight vectors begins to shine brightly. The noise to signal ratio for IMC and SIMC is independent of frequency below 2.5 Doppler widths, but then climbs sharply as the frequency is increased and the signal drops in the wing of the line. For SIMC with weight vectors, however, the noise to signal ratio drops rapidly with increasing frequency, maintaining a good statistical accuracy in the wing of the line. This happens because every photon carries spectral information deterministically in this method. This advantage can be very important if accurate problem output is required in the rarely sampled wing of the line.

When employing geometric zoning, with unbiased emission, the probability that a photon is born in the thin zones near the surface is quite small. Biasing allows for more sampling to occur in the thinner zones (where the upper atomic population is changing and the output spectrum is being influenced) and less in the larger zones (where very little is happening). The basic idea behind geometric zoning along with biasing is that regions that experience changes in upper atomic population are more important to development of the spectrum of transported photons. Therefore, they should be sampled more often in order to resolve finer structural details. This is accomplished by emitting a fixed 


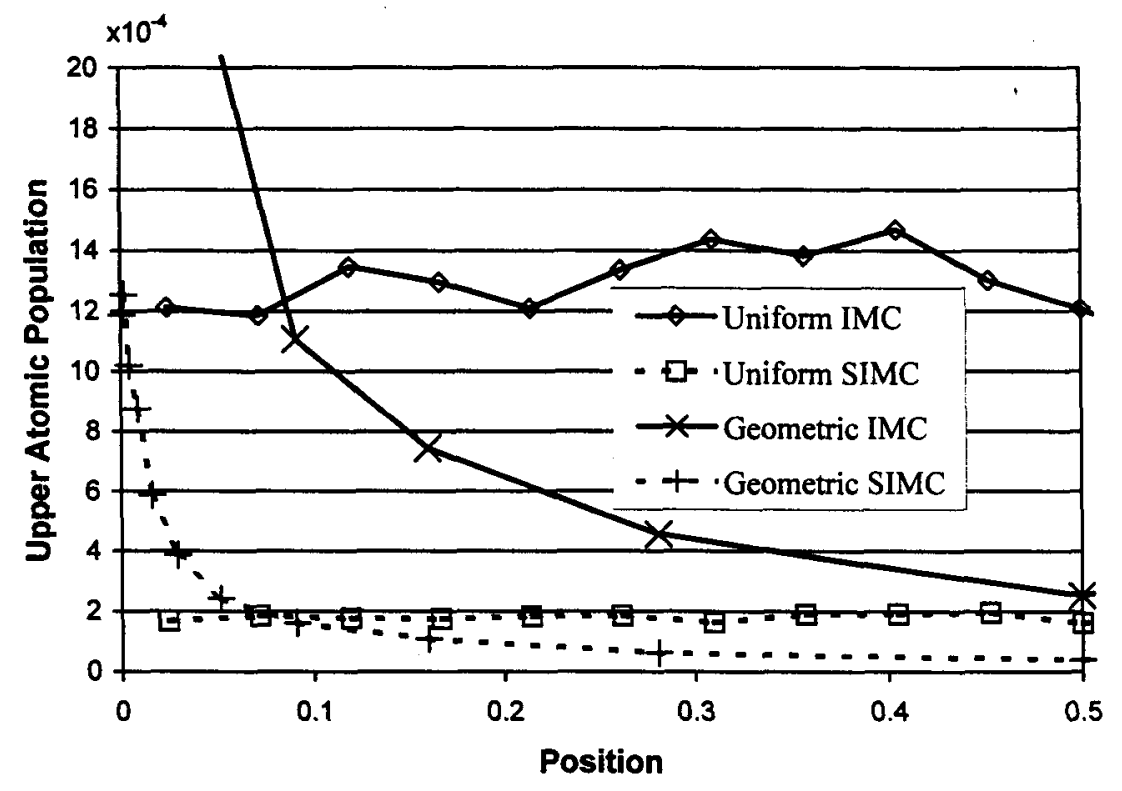

Fig. 3. Noise for Upper Atomic Population for Collisionally Pumped Trap Example

number of particles per zone, leading to a geometric weighting that favors emission near the surface.

As a second step in importance sampling, we add subzone biasing in which particles are more likely to be emitted closer to a zone's edge. As with the reasoning behind geometric zoning with an equal number of particles emitted per zone, subzone biasing places Monte Carlo photons closer to the interface of a zone so that they will more likely cross into another zone and contribute to radiative transfer between zones. Monte Carlo photons born deep within a zone, at line center, lose a large amount of weight through attenuation before reaching a boundary and are therefore less important.

The effects of biasing are shown in the noise to signal ratio of Fig. 6. Comparing to Fig. 5, all three codes have improved their noise significantly at line center, at the cost of the noise becoming worse far from line center. SIMC with weight vectors, however, provides a uniformly low noise to signal ratio for the complete frequency spectrum. As a note, the effect of subzone biasing is not apparent here as the frequency spectrum is developed in the thin zones near the surface. Subzone biasing improves the frequency spectrum of photons crossing an interface deep inside the slab. 


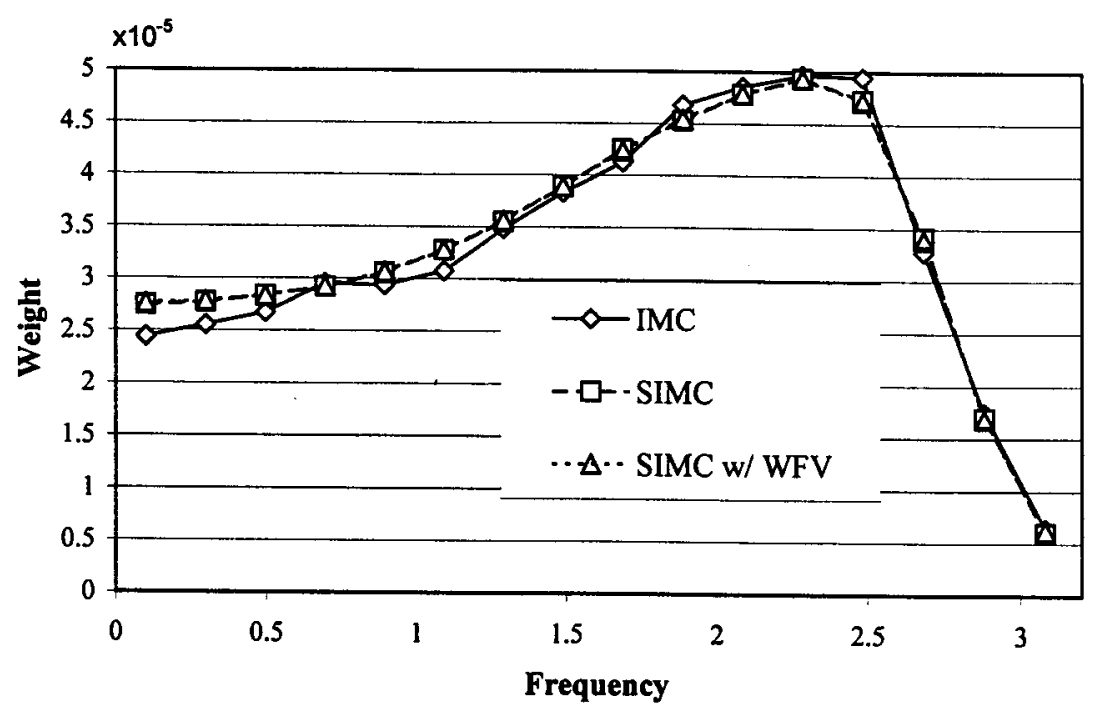

Fig. 4. Exit Frequency Distribution for Collisionally Pumped Trap Example

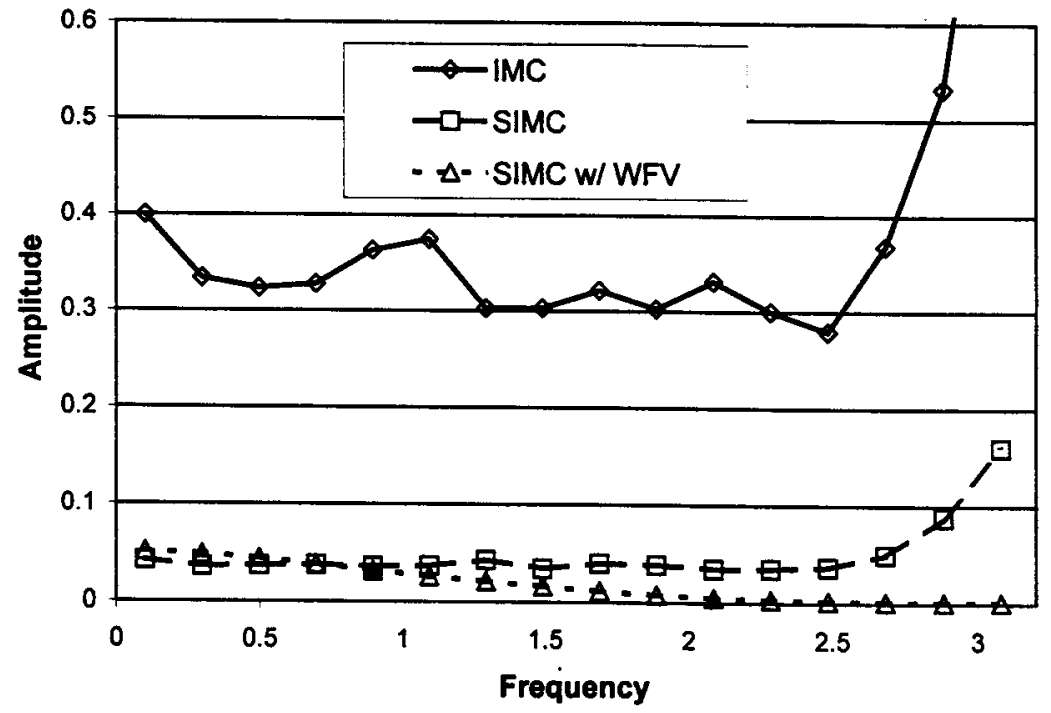

Fig. 5. Noise to Signal Ratio in the Emission Spectrum the Fully Biased Collisionally Pumped Trap Example

\subsection{Surface Heating Problem}

Our second problem is the line transport version of a slab with surface heating. Low intensity radiation impinges on the left surface of an optically thick slab 


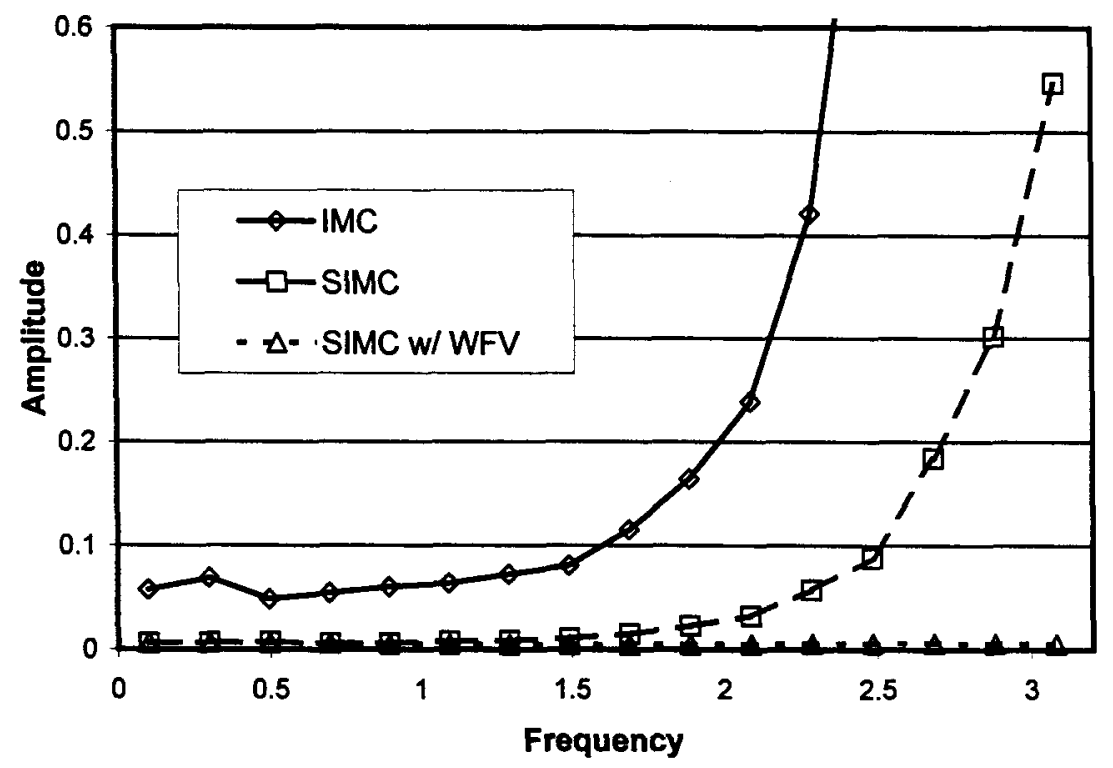

Fig. 6. Noise to Signal Ratio in the Emission Spectrum for Fully Biased Collisionally Pumped Trap Example

perpendicularly. The incoming beam is monochromatic in the wing of the line. As the beam penetrates the slab, photons are absorbed and reemitted with a spectrum given by the line profile. The slab is initially cold and has an initial optical depth of 100 at line center. The problem parameters are given in Table 2.

The incoming radiation heats the slab, first heating the surface and then causing it to emit radiation with a profile characteristic of the trapping problem presented in Sec. 3.1. The excitation then traverses the slab and transmission eventually occurs out the far side. This form of heating is interesting in that it clearly shows how IMC and SIMC can make systematic errors. The data in Figs. 7 and 8 is for time $=200$, achieving steady-state at the surface and extending to the middle of the slab, but is just short of steady-state for the transmitted radiation due to the long time to penetrate the slab. We vary the time step size and the number of zones, in order to examine the effect on the IMC and SIMC methods.

In Fig. 7, the upper level atomic population versus position, at time 200, for the first $20 \%$ of the slab is shown. The "Base IMC" and "Base SIMC" curves are shown for 21 equally spaced zones, and a time step size of 5 . Also shown is the result for the IMC algorithm, with the time step size reduced by a factor of 256 , and the result for the SIMC algorithm, with the zone size reduced 
Table 2

Physical Parameters for Surface Heating Problem

\begin{tabular}{cc}
\hline Parameter & Value \\
\hline$n(x, t=0)$ & 0 \\
$f(\mu, \nu, x, t=0)$ & 0 \\
$f(\mu>0, \nu, x=0, t)$ & $1 \times 10^{-5}$ \\
$f(\mu<0, \nu, x=1, t)$ & 0 \\
Voigt Parameter & 0.0 \\
$K_{12}$ & 170 \\
$K_{21}$ & 170 \\
$A_{21}$ & 3.3 \\
$C_{12}$ & 0 \\
$C_{21}$ & 0 \\
Incoming Frequency (Doppler Width) & 1.3 \\
\hline
\end{tabular}

by a factor of 10 . This refined zoning for the SIMC algorithm provides the correct solution for the problem, with the 21 zone IMC solution being close to correct in the sense of the average of the correct solution over the coarser zoning. The results for SIMC with 21 zones suffer from photon teleportation problems, as discussed in Sec. 2.5. The IMC algorithm is less sensitive to photon teleportation, as long as one does not refine the time step. When the time step for IMC is reduced by a factor of 256, it also suffers teleportation error, falling into agreement with SIMC for the 21 zone case. The IMC does well for a coarse zone and time step choice (apart from increased noise), but the solution moves in the wrong direction when the time step size is reduced.

In Fig. 8, we show the flux leaving the right hand side of the slab at time 200. All of the methods, and choices of zoning and time step size, neglecting noise, agree pretty well where significant energy is transported far from line center. Again, photon teleportation becomes a problem near line center if the time step for IMC is refined, or large zone sizes are used for SIMC. Note the very significant advantage for SIMC with weight vectors in the region where significant energy is transported.

The effects of photon teleportation error become clear when we examine the time dependent heating of a region near the surface of the slab (the region occupied by the first zone for the case of 21 equally spaced zones and average of the corresponding zones in the case of 210 zones) and the time dependent heating of a similar region in the middle of the slab. Again, IMC and SIMC with zone refinement give correct solutions. In Fig. 9, we show the surface heating as a function of time. In this case, teleportation error results in slower 


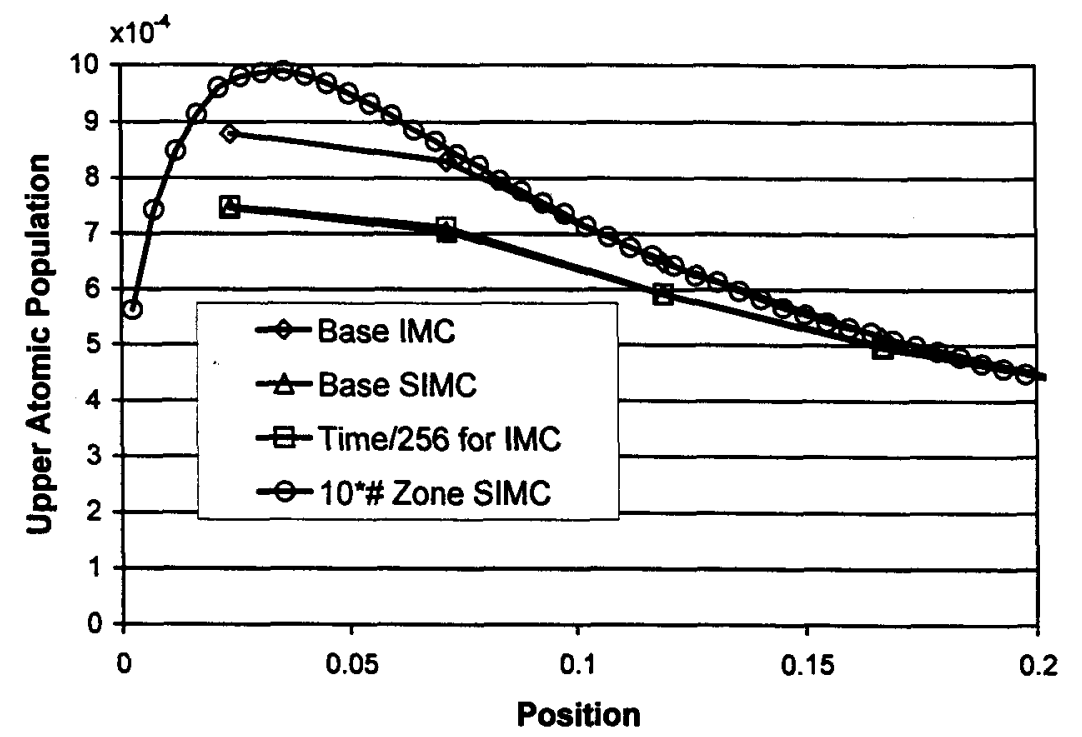

Fig. 7. Upper Atomic Population for the Surface Heating Example at Steady-State

heating and an overall colder region. In Fig. 10, we show the heating in the middle of the slab as a function of time. Accumulated photon teleportation error is overheating this region. This is consistent with the view that photon teleportation error moves energy through the material too quickly.

Overall, this example demonstrates that IMC is much more forgiving than SIMC, with respect to bad zoning. One must be careful, however, in becoming too reliant on this feature of IMC. IMC will suffer from photon teleportation error if the time step is refined, and the user may think that the solution is getting better rather than worse. Therefore, we have concluded that the convergence characteristics of IMC, in response to time step and zone refinement, are very poor and it is hard to know that one has a correct solution. SIMC, on the other hand, demonstrates rapid convergence in response to time step and zone refinement.

\subsection{Milne's Problem}

In our last test problem, we consider a localized source in the middle of the slab and an opacity that is independent of frequency. This approximation for the opacity, known as the grey approximation or Milne's problem [7], offers an analytical solution that can be used to check the accuracy of the numerical results. 


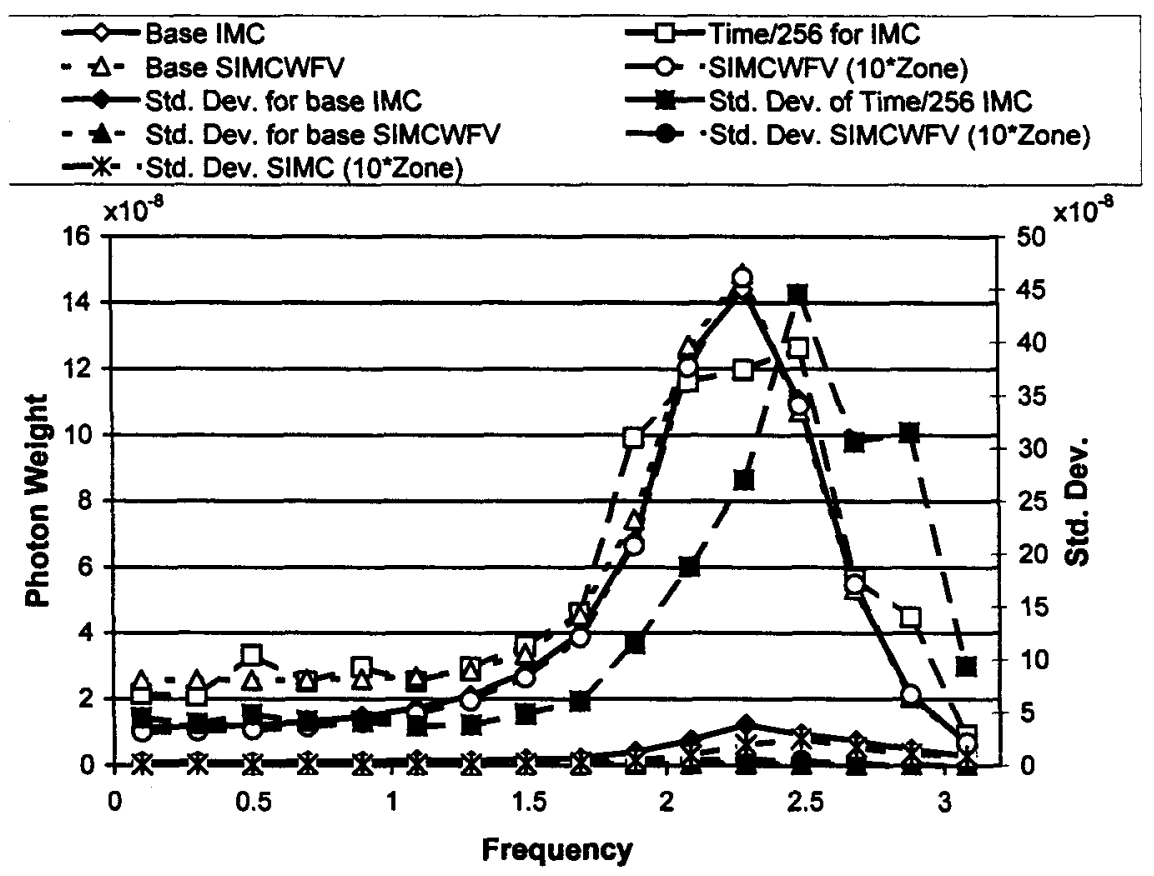

Fig. 8. Photon Frequency Distribution Leaving the Right Side of the Slab for Surface Heating Example

We simulate a grey slab by defining only one energy group of width $\Delta \nu$. The physical configuration of this problem can be seen in Fig. 11. We define a central zone to provide a source of collisionally pumped photons of width $L_{\text {exc. }}$. In this central zone, $C_{12}$ is to set a small value consistent with the approximations required for an analytical solution. The problem is set up with 21 equally spaced zones and the physical parameters specified in Table 3.

The analytical solution is provided in Appendix A. We cannot easily solve for $n$ close to the pumped region or near the edge of the slab since the boundary layers add additional complexity. However, the slope of $n$ far from the boundaries may be obtained analytically.

The predicted slope of $n$ versus position, equation (A-7) from the appendix, is $-1.7 \times 10^{-4}$. The results of IMC and SIMC have been plotted in Fig. 12. IMC and SIMC produce slopes of $-1.7 \times 10^{-4}$ and $-1.3 \times 10^{-4}$, respectively. Although SIMC has a lower noise figure than IMC, as seen in this graph, it produces the wrong slope. The directional dependence of photons, $\kappa$, as defined in equation (B-1) was found experimentally to be about 1.6. 


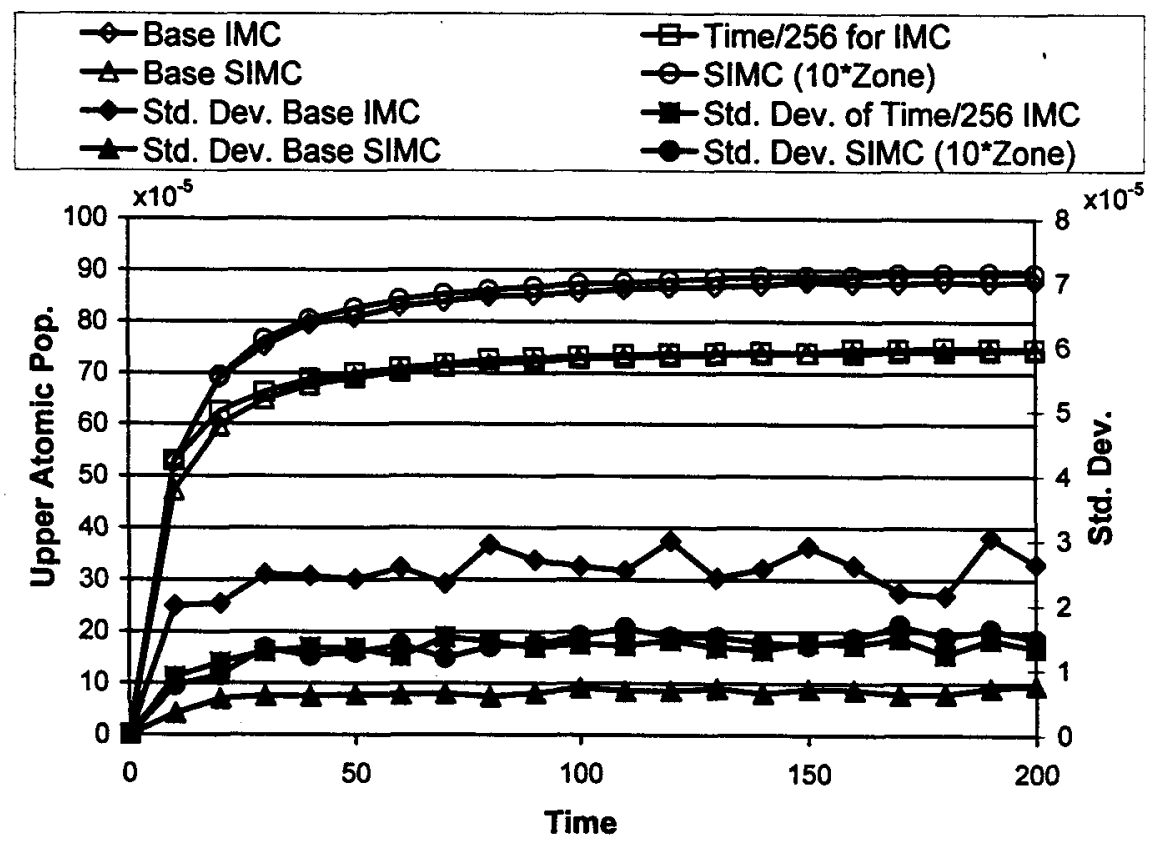

Fig. 9. Surface Heating as a Function of Time for Surface Heating Problem

Table 3

Physical Parameters for Milne's Problem

\begin{tabular}{cc}
\hline Parameter & Value \\
\hline$K_{12}$ & 170 \\
$K_{21}$ & 170 \\
$A_{21}$ & 3.3 \\
$C_{12}$ (Center Zone) & $1 \times 10^{-5}$ \\
$C_{12}$ (Other Zones) & 0.0 \\
$C_{21}$ & 0.0 \\
$L_{\text {exc }}$ & 0.0476 \\
$\Delta \nu$ & 6 Doppler Widths \\
\hline
\end{tabular}

This error for SIMC is due to the teleportation problem discussed in Sec. 2.5. When we refine the zoning by a factor of ten, which reduces the optical depth per zone, SIMC and IMC both agree with the predicted slope, $-1.7 \times 10^{-4}$. Attempts to use geometric zoning schemes, with thick zones in the middle of the uniform regions on the left and right sides of this problem, suffer from the 


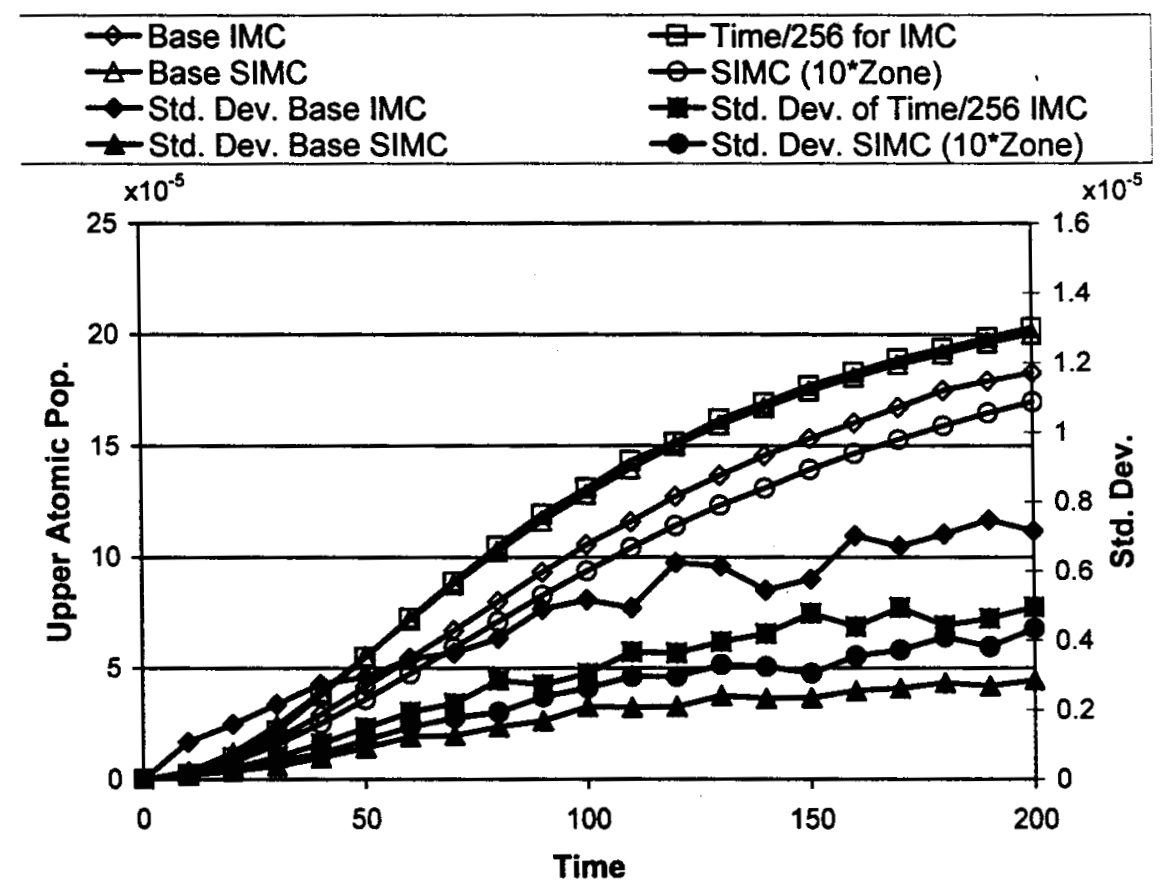

Fig. 10. Mid-slab Heating as a Function of Time for Surface Heating Problem

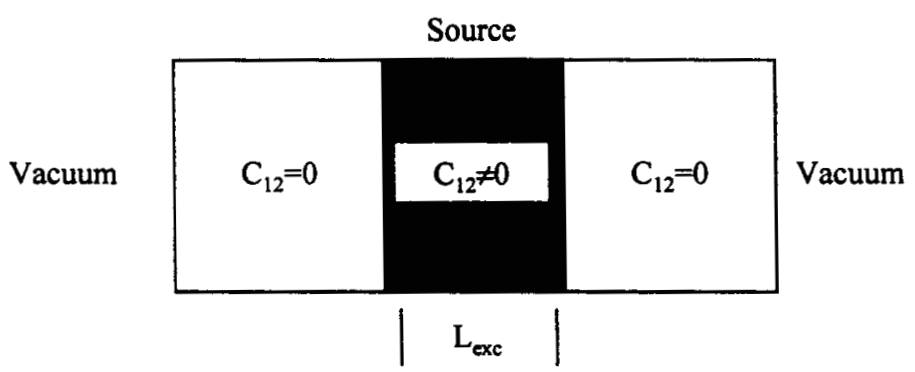

Fig. 11. Milne's Problem Layout

photon teleportation problems described previously.

Equation (B-5) from the appendix, displayed below, shows how teleportation error affects the predicted slopes for this problem.

$$
\frac{d n}{d x} \cong-\frac{3 F\left[K_{12}-\left(K_{21}+K_{12}\right) \bar{n}\right]^{2}}{A_{21} \Delta \nu^{2}} \frac{\tanh (\lambda / 2)}{\lambda / 2}
$$




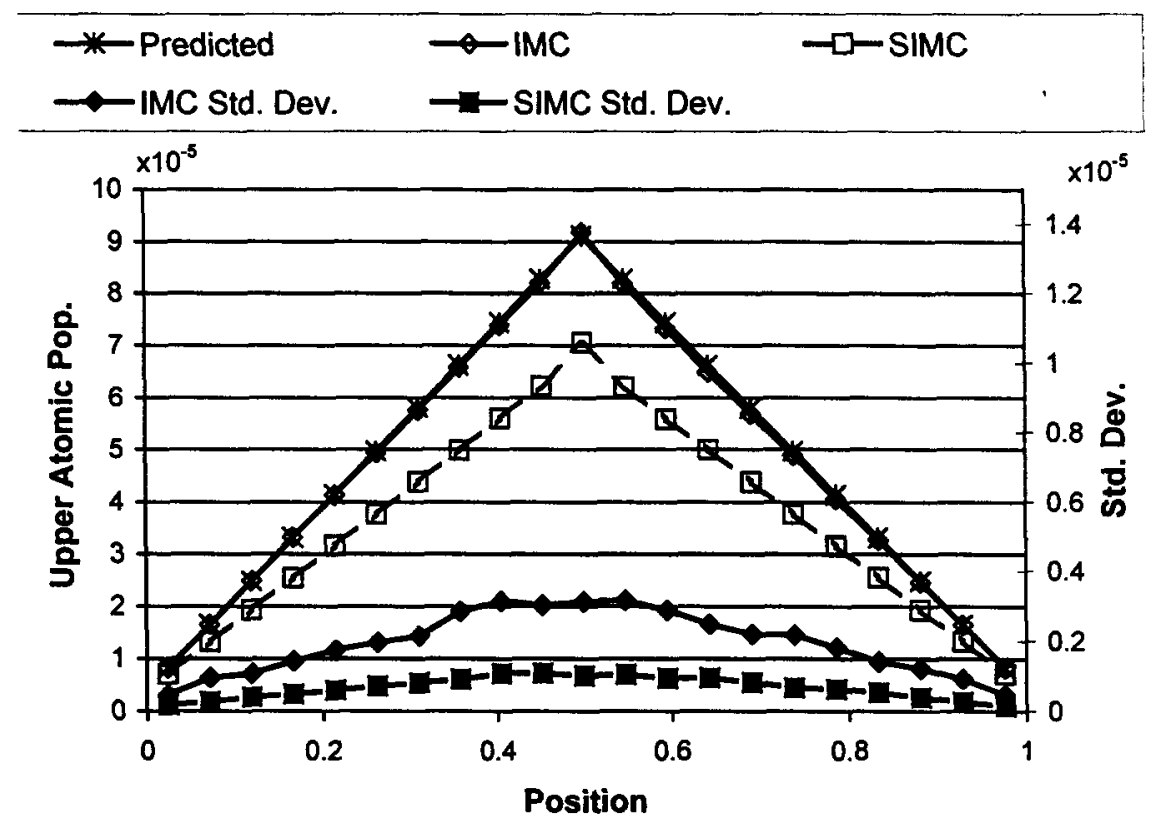

Fig. 12. Results for Milne's Problem

The multiplicative term, $[\tanh (\lambda / 2)] /(\lambda / 2)$, modifies the slope based on the optical depth per zone, $\lambda$. As the optical depth is decreased, $\lambda$ goes to 0 and the multiplicative term asymptotically approaches 1 ; so it converges to the correct solution. In the other limit as the optical depth is increased, the factor goes to 0 and reduces the computed slope.

In this example problem for SIMC, $\lambda$ is 2.16 (assuming the value of $\kappa$ to be 1.6) while a tenfold increase in zoning leads to $a$ of 0.216 . Since in the derivation of equation (B-5) we assumed $\lambda$ was much less than 1 , we cannot use it to double check the computed slope of the example problem. However, we can see that a $\lambda$ of 0.216 yields a $0.4 \%$ error. While not derived for the collisionally pumped trap problem, it does reinforce the observation that you need finer zoning in places of large slope while zones with little to no slope do not need as much refinement.

In the IMC method, the effective scattering reduces the absorption and this must be taken into account. The resulting equation is very similar to (B-5) with a minor change. Since the absorption cross section is $\hat{f} \sigma$ as given in equation (10), the absorption per zone is reduced by a factor of $\hat{f}$. This results 
in a prediction for the slope for IMC as

$$
\frac{d n}{d x} \cong-\frac{3 F\left[K_{12}-\left(K_{21}+K_{12}\right) \bar{n}\right]^{2}}{A_{21} \Delta \nu^{2}} \frac{\tanh (\hat{f} \lambda / 2)}{(\hat{f} \lambda / 2)}
$$

From this expression, we can see how IMC's effective scattering dampens the teleportation error. As $\hat{f}$ approaches 1 (corresponding to smaller time steps), the scattering contribution disappears and this equation approaches equation (B-5) and behaves as SIMC. As $\hat{f}$ decreases, the multiplicative term from above contributes less and less to the behavior of the slope. The limiting value of $\hat{f}$ is $\left(C_{12}+C_{21}\right) /\left(C_{12}+C_{21}+A_{21}\right)$ which represents the most that the teleportation error may be dampened.

\section{Conclusions}

In this paper, we have compared Implicit Monte Carlo (IMC) to the Symbolic Implicit Monte Carlo (SIMC) technique, using collisional pumping, the surface heating problem and the Milne problem as diagnostic applications. SIMC was also extended with a temporally persistent version of the weight vector approach of [5], demonstrating the value of weight vectors when accurate spectral information is desired. In addition to the numerical runs, partial analytical solutions were used to verify the accuracy of the Monte Carlo solutions and to point to the sources of systematic errors when they occurred.

An important result of this investigation is to demonstrate conditions where SIMC and IMC succeed and fail. In general, SIMC produces much lower noise for high opacity problems, with the spectral results using the weight vector approach being truly stellar, because the SIMC algorithm does not expend computer time performing non-physical effective scattering. This performance advantage comes at the price of increased sensitivity to teleportation error that results from high opacity per zone and from additional time needed to solve a linear system of equations with matrix size based on thê number of zones. Although teleportation error will also occur for IMC, the level of severity is moderated by the portion of the physical absorption that is rolled over into effective scattering. Finer zones are required for SIMC than are needed for $\mathrm{IMC}$ as a result.

While the effective scattering in IMC dampens the effect of teleportation error, it becomes highly subject to this problem when the time step is small. When effective scattering is small, sensitivity to teleportation error rises to a level equivalent to that of SIMC. This is a somewhat unfortunate situation for the IMC algorithm. Generally, we expect that computational results should 
improve as the time step is decreased. In IMC, the accuracy of results can become worse due to the increasing influence of teleportation error and the user must be very careful as a result.

The weight vector extension provides spectral information with a very good noise figure, especially for regions of frequency space that would be sampled with low probability in the line profile. Global results that are the result of frequency integrations do not benefit significantly from this extension (on scalar processors) due to the additional cost of computing exponentials for each element of the weight vector for each track the particle makes. Weight vectors have the advantage that the distance to the next zone has to be computed only once. On vector processors, employed in [5], where a vector of a dozen or so exponentials would take the same time as a single scalar exponential, the weight vector extension of SIMC does not require much additional time and improved algorithm performance is worthwhile even if spectral information is not required.

Geometric zoning, so that thin zones are used near transition regions, provides a significant improvement in the accuracy of the solution provided by SIMC. The length of tracks in the limit of large time step are controlled by the zoning in SIMC, and not by effective scattering. Careful zoning in SIMC, which inherently has a smaller execution time than IMC, improves the accuracy of the solution. Zones should be thin where the upper level atomic population varies rapidly in space. The strategy works well for SIMC if the error introduced by photon teleportation is carefully watched.

IMC can benefit from zone refinement, especially if small time steps are required to improve temporal resolution. However, a word of caution must be given here. The IMC algorithm can demonstrate poor convergence behavior due to teleportation error as the time step is reduced and we have actually seen results for IMC actually get worse when the time step is reduced independently of the zone size. IMC seems to be somewhat magical in that it delivers good results for coarse time steps and zoning, but its results can get worse in response to refinement if one is not careful. SIMC responds much more systematically to independent zone and time step refinements, with good convergence characteristics on both fronts.

Biasing significantly reduces the noise for SIMC, especially if the line center opacity is high. Biasing the spontaneous emission in favor of the thin zones near the surface of a transition region and in favor of thin subzones near the surface of a centrally located thick zone in cases of high line center opacity, can produce results of very high precision and low noise if one wants to examine the physics of a boundary layer. The IMC algorithm is less capable in this regard. 
In general, all of the methods examined in this paper have their advantages and disadvantages. The teleportation issue provides an advantage for IMC, providing relatively coarse zoning and time step sizes are adequate. If one wants to perform significant zone and time step refinement in order to produce high accuracy results, however, SIMC becomes the method of choice with its better convergence behavior. If spectral information is required in a high opacity region or a transition region, the SIMC method extended with weight vectors provides the best method.

If the problem of teleportation were eliminated, the one disadvantage of SIMC evaporates, and the method would become the method of choice. We will examine the possibility of accomplishing this, using a new formulation for the transport equation, in future work.

\section{Appendix}

\section{A. Upper Atomic Population Derivation for Milne's Example}

The slope of the upper atomic population may be derived by first starting with equations (5) and (6) and looking at just the central zone. The flux, $F$, is defined as usual,

$$
F=\int_{-1}^{1} d \mu \int_{\nu}^{\nu+\Delta \nu} d \nu \mu f(\nu, \mu ; x, t)
$$

Setting all derivatives with respect to time to zero and integrating the transport equation (5) over angle and frequency and then adding it to equation (6) gives

$$
\frac{\partial F}{\partial x}=C_{12}(1-n)
$$

Since this problem is symmetric, we can integrate this equation over the right half the central zone of width, $L_{\text {exc }}$, giving

$$
F=\int_{\frac{1}{2} \text { Slab }} d x C_{12}(1-n)=\int_{\frac{1}{2} L_{\text {exc }}} d x C_{12}(1-n) \cong \frac{C_{12}}{2}(1-\bar{n}) L_{\text {exc }}
$$

where $\bar{n}$ is the average upper atomic population over the source region. This gives the total number of excitations per time. For the rest of this problem, 
we are only concerned with the right hand side of the slab where $C_{12}$ is equal to 0 .

In order to get an analytical solution outside the pumped region, we notice that the flux is constant there and it is given by equation (A-3). Guided by the diffusion approximation, we now assume that that the radiation field is given by the form

$$
f(x, \mu)=B(x)+\mu g(x) .
$$

where $B(x)$ is an isotropic Planckian distribution,

$$
B(x) \equiv f_{0}(x)=\frac{n A_{21}}{2 c\left[K_{12}-\left(K_{21}+K_{12}\right) n\right]}
$$

and the second term is a $P_{1}$ distribution.

The flux can be found from equation (A-1),

$$
F=c \int_{-1}^{1} d \mu \int_{\nu}^{\nu+\Delta \nu} d \nu(B+\mu g)=\frac{2}{3} c \Delta \nu g
$$

which shows that $g$ is not a function of space. We also note that the line profile, $\phi$, must integrate over frequency to give 1 . Therefore it must be equal to the value $1 / \Delta \nu$. We now substitute $f$, from equation (A-4), into equation (5) and use the value of $B$ from equation (A-5) and the fact that $g$ does not vary in space. From this we get a relationship between $B$ and $g$ which may be used in equation (A-6) to obtain a value of $F$ for the left hand side of the slab. This $F$ must be equal to the $F$ from equation (A-3). Setting these two equal to each other and assuming that $n<1$, we obtain a solution for the slope of the upper atomic population:

$$
\begin{aligned}
\frac{d n}{d x} & =-\frac{3 F\left[K_{12}-\left(K_{21}+K_{12}\right) n\right]^{3}}{A_{21} K_{12} \Delta \nu^{2}} \\
& =-\frac{3}{2} \frac{C_{12}}{A_{21}} \frac{\left[K_{12}-\left(K_{21}+K_{12}\right) n\right]^{3}}{K_{12} \Delta \nu^{2}} L_{e x c}(1-\bar{n}) \\
& \cong-\frac{3}{2} \frac{C_{12}}{A_{21}} \frac{K_{12}^{2}}{\Delta \nu^{2}} L_{e x c}
\end{aligned}
$$

B. Teleportation Error Derivation 
The difficulity in setting up a problem lies in knowing how fine the mesh must be to reduce teleportation error. For this problem, we can define a parameter to represent the optical depth per zone as given in the following equation:

$$
\lambda=\frac{\kappa L_{z o n e}\left[K_{12}-\left(K_{21}+K_{12}\right) \bar{n}\right]}{\Delta \nu} \cong \frac{\kappa L_{z o n e} K_{12}}{\Delta \nu} .
$$

where $\kappa$ is factor that takes into account directional dependence of the photons.

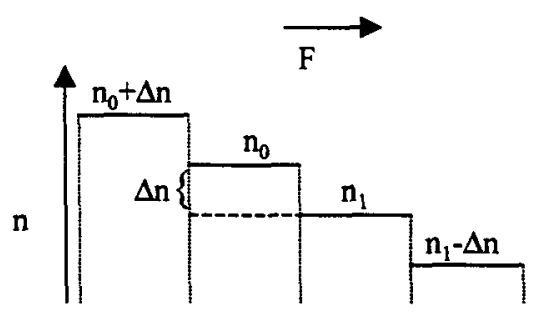

Fig. B-1. Zoning Diagram for Milne's Problem

This problem has been discretized into equal sized zones with the assumption that the upper atomic population, $n$, is constant across a zone. Since we have focused on the right side, $n$ can be represented by a step function with steps at each multiple of $\lambda$ as shown in Fig. B-1. In this figure, $\Delta n$ is equal to $n_{0}-n_{1}$. We are interested in computing $F$ at the midpoint in terms of $\lambda$ and then relate this to the slope of the upper atomic population. Since we wish to study the effects of small optical depth per zone length, we assume $\lambda$ is much less than one. Doing so allows us to approximate the angle integrated photon density distribution by integrating the emission in each zone and differencing the flux from the right and left as

$$
\begin{aligned}
F= & \frac{1}{3} \Delta \nu c\left\{\left[\left(1-e^{-\lambda}\right) B\left(n_{0}\right)+\left(e^{-\lambda}-e^{-2 \lambda}\right) B\left(n_{0}+\Delta n\right)+\cdots\right]\right. \\
& \left.-\left[\left(1-e^{-\lambda}\right) B\left(n_{1}\right)+\left(e^{-\lambda}-e^{-2 \lambda}\right) B\left(n_{1}-\Delta n\right)+\cdots\right]\right\} .
\end{aligned}
$$

The steady-state solution, $B$, has slightly changed from its form in equation (A-5) to its new form as

$$
B(n)=\frac{n A_{21}}{2 c\left[K_{12}-\left(K_{21}+K_{12}\right) \bar{n}\right]}
$$

The bracketed terms in equation (B-2) can be summed to get the following 
solution for $F$ :

$$
\begin{aligned}
F & =\frac{1}{3} \Delta \nu c \frac{A_{21}}{2 c\left[K_{12}-\left(K_{21}+K_{12}\right) \bar{n}\right]}\left\{n_{0}-n_{1}+\frac{2 e^{-\lambda}}{1-e^{-\lambda}} \Delta n\right\} . \\
& =\frac{1}{3} \Delta \nu c \frac{A_{21}}{2 c\left[K_{12}-\left(K_{21}+K_{12}\right) \bar{n}\right]} \frac{1}{\tanh (\lambda / 2)}
\end{aligned}
$$

The slope can now be approximated by using the definition of $\lambda$ from equation (B-1) along with equation (B-5) to arrive at

$$
\frac{d n}{d x} \cong-\frac{\Delta n}{L_{\text {zone }}}=-F \frac{3\left[K_{12}-\left(K_{21}+K_{12}\right) \bar{n}\right]^{2}}{A_{21} \Delta \nu^{2}} \frac{\tanh (\lambda / 2)}{\lambda / 2} .
$$

\section{References}

[1] F. Paletou, Transfert de Rayonnement: Méthodes Itératives, C. R. Acad. Sci. Paris, t. 2, Série IV, 6, 885-898 (2001).

[2] M. Noort, I. Hubeny, and T. Lanz, Miltidimensional Non-LTE Radiative Transfer. I. A Universal Two-Dimensional Short-Characteristic Scheme for Cartesian, Spherical, and Clyindrical Coordinate Systems, Astrophysical Journal, 568, 1066-1094 (2002).

[3] E. D. Brooks III and J. A. Fleck, Jr., An Implicit Monte Carlo Scheme for Calculating Time-Dependent Line Transport, Journal of Computational Physics, 67, 59-72 (1986).

[4] E. D. Brooks III, Symbolic Implicit Monte Carlo, Journal of Computational Physics, 83, 433-446 (1989).

[5] T. N'Kaoua, Solution of the Nonlinear Radiative Transfer Equations by a Fully Implicit Matrix Monte Carlo Method Coupled with the Rosseland Diffusion Equation Via Domain Decomposition, SIAM Journal Scientific and Statistical Computing, 12 505-520 (1991).

[6] T. N'Kaoua and R. Sentis, A New Time Discretization for the Radiative Transfer Equations: Analysis and Comparison with the Classical Discretization, SIAM Journal on Numerical Analysis, 30 733-748 (1993).

[7] D. Mihalas, Stellar Atmospheres, W. H. Freeman and Company, San Francisco, 1978.

[8] A. Jennings, Matrix Computation for Engineers and Scientists, WileyInterscience, New York, 182 (1977).

[9] D. Mihalas and R. I. Klein, On the Solution of the Time-Dependent InertialFrame Equation of Radiative Transfer in Moving Media to $O(v / c)$, Journal of Computational Physics, 46, 97 (1982). 
[10] M. L. Adams and P. F. Nowak, Asymptotic Analysis of a Computational Method for Time- and Frequency-Dependent Radiative Transfer, Journal of Computational Physics, 146, 366-403 (1998).

[11] G. L. Olson, L. H. Auer, and M. L. Hall, Diffusion, $P_{1}$, and Other Approximate Forms of Radiation Transport, Journal Quantitative Spectroscopy and Radiative Transfer 64, 619-634 (2000).

[12] J. E. Morel, T. A. Wareing and K. Smith, A Linear-Discontinuous Spatial Differencing Scheme for $S_{n}$ Radiative Transfer Calculations, Journal of Computational Physics, 128, 445-462 (1996).

[13] D.Balsara, Fast and Accurate Discrete Ordinates Methods for Multidimensional Radiative Transfer. Part I, Basic Methods, Journal Quantitative Spectroscopy and Radiative Transfer, 69, 671-707 (2001).

[14] J. E. Morel, Diffusion-Limit Asymptotics of the Transport Equation, the $P_{1 / 3}$ Equations, and Two Flux-Limited Diffusion Theories, Journal Quantitative Spectroscopy and Radiative Transfer, 65, 769-778 (2000).

[15] J. A. Fleck and E. H. Canfield, Random Walk Procedure For Improving The Computational Efficiency Of The Implicit Monte Carlo Method For Nonlinear Radiation Transport, Journal of Computational Physics, 54, 508-523 (1984). 